\title{
Le texte épistolaire comme source historique : les lettres de Marie Dorval à Alfred de Vigny (1833-1837), tableau de la vie théâtrale en province
}

Parmi les documents offerts à l'historien de la vie théâtrale en province figurent, aux côtés de la presse, des archives des théâtres ou des mémoires d'artistes, ces témoignages sur le vif que constituent les correspondances d'acteurs. Celles-ci sont loin d'être toutes conservées, recueillies et publiées comme ont pu l'être, pour citer deux exemples célèbres, les lettres de Marceline Desbordes-Valmore ou de Rachel $^{1}$ : on peut rêver aux trésors épistolaires enfouis dans quelques archives publiques ou fonds privés, en attente de lecture, de regroupement, de classement, de datation ou d'annotation. C'est à semblable recherche que cette communication, fondée modestement sur des lettres déjà connues et publiées, souhaite encourager la petite communauté rassemblée par Sophie Leterrier.

Parmi ces correspondances d'acteurs, celle de Marie Dorval jouit d'un privilège éditorial dû non seulement à la carrière exceptionnelle de la reine du drame romantique et moderne, mais aussi au prestige littéraire de deux de ses correspondants principaux : Alfred de Vigny et George Sand ${ }^{2}$. Les lettres à Vigny, qui nous intéressent ici, ont été recueillies et publiées dès 1942 à la $n r f$, par Charles Gaudier ${ }^{3}$. Cette édition a été depuis parfaite, complétée et corrigée par la publication entre 1989 et 1997, aux Presses Universitaires de France, de la Correspondance d'Alfred de Vigny, édition réalisée sous la direction de Madeleine Ambrière ${ }^{4}$. Les lettres adressées par Dorval à l'amant-poète y sont reprises et annotées avec toute la rigueur et la précision scientifiques nécessaires. Précisons d'emblée que, pour le lecteur d'aujourd'hui, cet échange épistolaire tourne au monologue, du fait de la destruction de la plus grande partie des lettres de Vigny à Dorval. Les missives de Dorval n'en sont souvent que plus pathétiques, série d'appels qui nous semblent sans réponse. Une petite déception attend aussi les modernes lecteurs que nous sommes : à l'instar de Charles Gaudier, Madeleine Ambrière corrige l'orthographe des documents originaux et nous prive ainsi d'un contact plus étroit avec la langue de Dorval. Seule une édition diplomatique rendrait l'orthographe originelle et même la graphie de celle qui déclare à Vigny : " Je voudrais avoir une jolie écriture comme celle de vos femmes du monde, une écriture à l'anglaise ${ }^{5} »$. La matérialité de la lettre fait donc défaut: la couleur de l'encre, la texture du papier, l'occupation de l'espace, la présence d'une enveloppe ou le recours au pliage, autant de signes que l'on aimerait pouvoir apprécier sensuellement. À défaut de toucher, voir, humer le document original, l'on ne peut que rêver à telle lettre de Vigny trop aérée aux yeux de son

\footnotetext{
${ }^{1}$ Citons : Lettres de Marceline Desbordes à Prosper Valmore, publiées par Boyer d'Agen, Paris, éditions de la Sirène, 1924, 2 vol. ; Rachel d'après sa correspondance par Georges d'Heylli, Paris, Librairie des Bibliophiles, 1882 ; Hector Fleischmann, Rachel intime d'après ses lettres d'amour et des documents nouveaux, Paris, Fasquelle, 1910.

${ }^{2}$ Avant la célèbre édition par Georges Lubin de la correspondance de George Sand, les lettres de Sand et Dorval ont été éditées par Simone André-Maurois, avec une préface d'André Maurois, dans la collection nrf de Gallimard, en 1953.

${ }^{3}$ Marie Dorval, Lettres à Alfred de Vigny, recueillies et présentées par Charles Gaudier, Paris, Gallimard, nrf, 1942.

${ }^{4}$ C'est l'édition utilisée ici : Correspondance d'Alfred de Vigny, sous la direction de Madeleine Ambrière (Centre de Recherche, d'Etude et d'Edition de Correspondances du XIX ${ }^{\mathrm{e}}$ siècle de l'Université de ParisSorbonne), PUF, 1989-1997, tomes 2 (août 1830-septembre 1835) et 3 (septembre 1835-avril 1839). Nous renvoyons désormais à cette édition par l'abréviation Corr. suivie des références de lieu, de date (entre crochets pour les mentions qui ne sont pas de la plume de Dorval), de tome et de page(s).

${ }^{5}$ Corr., [Rouen] Jeudi soir [29 août 1833], t. 2, p. 277.
} 
amante, toujours travaillée par un amour dévorant : « tu laisses du papier blanc ${ }^{6}$ », reproche-telle à son correspondant, tant la saturation du papier est pour elle gage de passion.

Ce déficit de matérialité est compensé par la scientificité de l'édition. L'annotation savante et précise d'Ambrière et de son équipe éclaire toutes les références, essentiellement théâtrales, de ces lettres, transformées en un matériau de premier ordre pour le travail sur la vie de province à l'époque romantique. Reste à savoir comment utiliser ce matériau : faut-il y voir une mine de renseignements et traiter ces lettres comme des documents où puiser à pleines mains un savoir prêt à se constituer ? Ou s'agit-il de les lire aussi comme des textes et comme des discours? Il s'agit non seulement de représentations d'une existence vécue, portées par une puissante subjectivité, mais aussi de lettres obéissant à une visée pragmatique - donner à voir, combler l'absence, séduire, rassurer, prouver sa passion, se montrer digne muse du poète, agir de loin sur le monde. C'est à ce double régime de lecture - du document, de la lettre, de la lettre devenue document - que je voudrais ici me livrer.

Je ne reviendrai pas, dans le temps de cette communication, sur les données biographiques, bien connues, de la comédienne ${ }^{7}$. Rappelons seulement que Dorval est issue, selon l'expression de Francis Ambrière, du «prolétariat comique des troupes ambulantes ${ }^{8}$ ». Née en 1798 de parents comédiens, elle monte sur les planches à quatre ans et ne met provisoirement fin à des tournées provinciales incessantes que vingt ans plus tard, en 1822, avec ses débuts au Théâtre de la Porte Saint-Martin. Le retour à la vie de comédienne ambulante au début des années 1830 doit sans doute beaucoup à quelque appel de la route chez celle dont la famille, digne d'un « roman comique », était vouée à l'errance perpétuelle.

Mais les tournées de plus en plus longues et lointaines en province sont surtout dues à des causes matérielles et institutionnelles : Dorval court le cachet loin de Paris car elle est endettée, poursuivie par les créanciers, et, de plus, rejetée des premières scènes parisiennes occupées par Mlle Mars ou Mlle George. Cette dernière a ainsi créé à l'Odéon en 1831 le rôle-titre de la Maréchale d'Ancre, que Vigny aurait aimé confier à sa nouvelle amante, Marie Dorval, la " première tragédienne existante » selon lui ${ }^{9}$. Mais Dorval est alors engagée à la Porte Saint-Martin où elle crée l'Antony de Dumas et Marion de Lorme de Hugo. Deux ans après, c'est encore Mlle George qui colonise les rôles nouveaux, cette fois à la Porte SaintMartin ; par ce monopole, elle contraint Dorval à rompre son contrat, le 5 juin 1833. Les cinq années 1833-1837, retenues pour cet exposé, voient dès lors Dorval courir de théâtre en théâtre sur les routes de province, forcée de différer sans cesse son retour à Paris où aucun théâtre ne semble véritablement l'attendre. Seule la première moitié de l'année 1835 replace provisoirement Dorval, grâce à l'appui de Vigny et Hugo, en haut de l'affiche parisienne, avec Chatterton puis Angelo à la Comédie-Française. Le début de l'année 1838, au terme de la période ici étudiée, offrira encore à Dorval un retour sous forme de revanche à la ComédieFrançaise pour des reprises d'Hernani, d'Angelo, de Marion Delorme. C'est finalement à la disgrâce de la reine des boulevards, tragédienne éclose sur la scène du mélodrame, régulièrement tenue à la lisière des théâtres principaux, que nous devons cette correspondance exceptionnelle, introduction indispensable à toute étude de la vie théâtrale en province dans le premier XIX ${ }^{\mathrm{e}}$ siècle $^{10}$.

\footnotetext{
${ }^{6}$ Ibid.

${ }^{7}$ Voir sur ce point le très riche ouvrage de Francis Ambrière, Mademoiselle Mars et Marie Dorval au théâtre et dans la vie, Paris, éditions du Seuil, 1992.

${ }^{8}$ Francis Ambrière, Le Siècle des Valmore, Editions du Seuil, 1987, t. I, p. 489. Nous renvoyons désormais à cette édition par l'abréviation Corr.

${ }^{9}$ Voir l'édition des Euvres complètes d'Alfred de Vigny, tome I, « Poésie, Théâtre », par François Germain et André Jarry, Paris, Gallimard, nrf, coll. « Bibliothèque de la Pléiade », 1986, p. 1443.

${ }^{10}$ « Le tableau de la vie des théâtres de province, esquissé de ville en ville et de jour en jour, les récits de la réception du drame romantique dans une province souvent hostile, voilà d'ailleurs un des intérêts majeurs de cette très belle correspondance » note Madeleine Ambrière, op. cit., t. 3, p. 8 .
} 


\section{Cinq années en « zig-zag »}

Présenter et situer ces cinq années de tournée provinciale de Dorval est fastidieux. Alfred de Vigny écrit à son amante, en septembre 1835, qu'il vient de découvrir grâce au journal le Vert-Vert que la comédienne est à Douai : "Que tes zig-zag sont difficiles à suivre, mon ange, comme tu es longtemps à courir ${ }^{11} »$. Pour nous aussi, il est difficile de suivre Dorval dans les méandres de ses tournées dont les étapes sont recensées lettre après lettre. L'année 1833 voit ainsi la comédienne se faire applaudir à Reims (24 mars- $1^{\mathrm{er}}$ avril), Compiègne, Soissons, Laon, Saint-Quentin (8 juillet-8 août), Rouen (20 août- 8 septembre), Cambrai et Arras (14 septembre-7 octobre), Le Havre (21 octobre-6 novembre), Rouen encore (23 décembre-16 janvier 1834). Cet éloignement de la capitale et de son amant entraîne une prolifération de lettres, toutes chargées de susciter la présence symbolique de celui dont elle emporte en voyage quelques objets fétiches: «J'emporte avec moi tous tes portraits, tes cheveux, des lettres, ta bourse, ton petit bonnet ${ }^{12} »$. Mais cette première année de séparation annonce d'autres voyages, plus frénétiques encore dans leur rythme, dans les années 1836-37, après une pause relative en 1834-35. L'année 1834 est essentiellement marquée, sur le plan des tournées, par un séjour à Bordeaux de fin janvier à fin février, où une rage de dents empêche Dorval de jouer : «La machine à argent ne va plus », écrit-elle à Vigny en janvier ${ }^{13}$. L'année 1835 est quant à elle marquée par la création de Chatterton à la Comédie-Française, le 12 février, puis, le 28 avril, par la première d'Angelo tyran de Padoue. En revanche, la seconde partie de l'année 1835 est occupée par une nouvelle tournée dans le nord : Lille (23 juillet-3 août), Boulogne (3-12 août), Arras (13-21 août), Dunkerque (22-30 août), la Belgique en septembre, puis Douai (4-12 octobre). Dorval enchaîne aussitôt avec une tournée dans l'ouest : Nantes (23 octobre-14 novembre), Brest (19-30 novembre), Lorient, sa ville natale (1-11 décembre), Nantes à nouveau (12-14 décembre). Puis l'année 1836 voit le centre de gravité des tournées se déplacer vers l'est et le sud : avec sa "petite charrette roulante ${ }^{14}$ », accompagnée de son cocher Jacques et de sa femme de chambre Victorine, Dorval visite Dijon puis Chalon-sur-Saône en juin et début juillet, Bourg-en-Bresse en juillet, Marseille du 23 juillet au 27 octobre, puis Lyon (29 octobre-3 décembre), Saint-Étienne (3-6 décembre), Avignon et Nîmes dans les derniers jours de l'année. La tournée dans le sud se poursuit au début de l'année 1837 : Toulouse, puis Béziers (8-9 mai), Narbonne et Carcassonne (10-19 mai), Béziers encore (21-22 mai), Pézenas, Montpellier (28 mai), Alès (30-31 mai), Sète, Arles début juin. Ce n'est qu'à la fin du mois de juin 1837 que Dorval retrouve Paris, mettant fin à une tournée entamée un an plus tôt. La halte est de courte durée : le voyage reprend de fin juillet à mi-octobre, à Troyes, Compiègne, Amiens, Caen, et de nouveau Rouen. L'énumération s'arrêtera là. La lecture des lettres qui jalonnent ces incessants voyages nous laisse dans un trouble voisin de celui que décrit Dorval depuis Toulouse en février 1837 : «Je confonds tout. J'oublie souvent d'où je viens et où je vais ${ }^{15} \ldots$ ». Mais l'intérêt historique de cette correspondance réside justement dans la diversité des lieux visités, dans la variété des théâtres et des troupes rencontrés. Ce corpus offre un tableau foisonnant de la vie des arrondissements théâtraux aux quatre points cardinaux de la France louis-philipparde.

\footnotetext{
${ }^{11}$ Corr., Mercredi 16 [septembre 1835], t. 3, p. 25.

${ }^{12}$ Corr., [Dimanche 24 mars 1833], t. 2, p. 234.

${ }^{13}$ Corr., [Bordeaux] Mardi [28 janvier 1834], t. 2, p. 305.

${ }^{14}$ Corr., Sur le chemin de Villeneuve-le-Roi, mardi [14 juin 1836], 9 h. du matin, t. 3, p. 93.

${ }^{15}$ Corr., Toulouse, 3 février 1837, t. 3, p. 218.
} 


\section{Splendeurs et misères des théâtres de province}

Le « roman comique » de Marie Dorval vaut d'abord pour ces infiniment petits de la vie théâtrale en province ${ }^{16}$ appelés à se déployer dans notre imagination. Les tribulations sur les chemins mal entretenus où la voiture part en morceaux alimentent les lettres de leurs multiples péripéties. L'on découvre ville après ville, jour après jour le même rituel, fixé dès la première tournée à Reims : «Je fais mes caisses pour le soir et je joue deux pièces. A $11 \mathrm{H}$. je fais mes comptes de la recette avec le directeur et sa femme - nous faisons des paquets de contre-marques jusqu'à minuit ${ }^{17}$ ». L'on pénètre, non sans voyeurisme, dans l'intimité de la comédienne pour partager la fébrilité de chaque soir de représentation, comme en cette chambre d'hôtel de Rouen où Dorval évoque « [...] des caisses à défaire, des robes à garnir, l'arrangement de tout cela, la peur, une peur horrible pour le soir $[\ldots]^{18} \gg$. L'on découvre enfin les «trucs » de tout acteur, entre médecine et superstition, comme ces fumigations de sureau pour améliorer la voix ${ }^{19}$.

La correspondance d'artiste éclaire surtout ce que laissent dans l'ombre les comptes rendus des journaux : le travail de répétition, la part prise par l'acteur parisien en tournée dans la mise en scène de la pièce. Dorval s'improvise dans chaque nouvelle ville régisseur, décoratrice et même chef d'orchestre : «Je mets tout le monde en scène, je règle les décors. Et on compose la musique séance tenante d'après mes indications ${ }^{20} »$. Le jugement sur les membres des troupes d'arrondissement est généralement sans concession : ces acteurs de troisième zone, recrutés par des directeurs peu regardants ou forcés de faire avec ce qu'ils trouvent, sont écrasés par la présence à leur côté de la vedette, cette grande actrice qui les a pourtant fait répéter et a tenté de leur transmettre son art. À propos de l'acteur Alexandre dans Antony, Dorval, toute puissante et rayonnante à Rouen, déclare : "C'est un acteur très médiocre qu'on supporte mais qui ne pourra pas rester - mon séjour ne lui fera pas de bien. Je le tue un peu avec mon naturel. Que je suis modeste ${ }^{21}$ ! ». Les portraits tracés par la plume acérée de Dorval, douée pour la caricature, sont souvent cruels. Ainsi de ses confrères dijonnais : ces «pauvres acteurs» sont d'une totale «ignorance», ne possèdent «pas la moindre distinction», n'ont «pas le moindre sentiment de 1 ' $\operatorname{art}^{22} »$. Au pire, ils sont «stupides », adjectif suivi sur la lettre de trois points d'exclamation ${ }^{23}$. Cette médiocrité est causée par l'impréparation des comédiens : à Dijon, pour Antony, les acteurs ont eu leur rôle « de la veille »; l'acteur principal « a appris son rôle dans la diligence » alors qu'il arrivait en catastrophe pour remplacer son collègue jeté en prison - il avait harangué le public à la manière du républicain Bocage ${ }^{24}$. Ce dernier n'est d'ailleurs pas mieux traité par Dorval que les obscurs comédiens ambulants : à Chalon où il fait partie de la deuxième troupe de l'arrondissement, Bocage joue « les bras pendants avec l'air aussi ennuyé qu'ennuyant ${ }^{25} »$. Il faut dire que les deux troupes sont en rivalité directe, artistique et politique, puisque les directeurs, Bousigue et Chauloux, sont respectivement royaliste et républicain. Et Dorval de dénoncer avec virulence un complot, ourdi par «la plus ignoble racaille de républicains

\footnotetext{
${ }^{16}$ On trouvera aussi une riche information dans l'ouvrage d'Anne Martin-Fugier, Comédienne. De Mlle Mars à Sarah Bernhardt, Paris, éditions du Seuil, 2001.

${ }^{17}$ Corr., [Reims] Samedi 30 [mars 1833] 1 h. du matin, t. 2, p. 236.

${ }^{18}$ Corr., [Rouen] Mercredi 21 août [1833] 11 h. 1/2 du soir, t. 2, p. 265.

${ }^{19}$ Corr., Dijon, samedi 18 juin [1836] 8 [h.] 1/2 du matin, t. 3, p. 96.

${ }^{20}$ Corr., [Reims] Samedi 30 [mars 1833] 1 h. du matin, t. 2, p. 236.

${ }^{21}$ Corr., [Rouen] Jeudi soir [29 août 1833], t. 2, p. 276.

${ }^{22}$ Corr., Dijon, samedi 18 juin [1836] 8 [h.] 1/2 du matin, t. 3, p. 96.

${ }^{23}$ Corr., Lyon, ce 29 [octobre 1836]. Samedi, t. 3, p. 170.

${ }^{24}$ Corr., Dijon, 15 [juin 1836]. Mercredi, t. 3, 95.

${ }^{25}$ Corr., Toujours jeudi [23 juin 1836], Chalon 10 h. du soir, t. 3, p. 104.
} 
d'estaminets que Bocage a ameutés contre mon directeur à moi qui l'a obligé de céder la place $^{26} \gg$.

La correspondance fourmille aussi de renseignements sociologiques et moraux sur le public, replacé dans les conditions matérielles des représentations. Ce tableau est fortement contrasté, Dorval descendant au plus bas et s'élevant au plus haut de la hiérarchie des villes de province. Au plus bas, durant ces années de galère théâtrale pour la comédienne, se trouve l'infâme ville de Chalon-sur-Saône, " ville odieusement laide, bêtement laide » où la troupe s'installe alors que la foire bat son plein : «me voici à la foire au milieu des polichinelles, des éléphants, des trompettes, des chiens savants et des chevaux de Franconi ${ }^{27} \gg$. Parmi cette foire, Dorval a l'impression de chuter définitivement de son piédestal ; elle n'est plus la tragédienne moderne célébrée par Vigny, mais une misérable saltimbanque dans un sordide spectacle de curiosités : «Ce brave public n'était pas en très grand nombre ; il est vrai que j'ai à lutter contre beaucoup d'animaux féroces et bêtes domestiques. Je me sens un peu humiliée et je ne puis m'empêcher de croire que je fais partie de la ménagerie ${ }^{28} »$. À l'autre extrémité de la hiérarchie des villes visitées se trouvent Marseille et son grand théâtre où, enfin, Dorval « fait de l'argent»: «Nous avons joué Chatterton devant une chambrée magnifique, devant la grande duchesse de Toscane et des princesses ${ }^{29} \ldots$ » s'exclame la comédienne ravie et émerveillée, tenant enfin sa revanche sur l'humiliation vécue. De même, à Toulouse, Dorval révèle triomphalement à Vigny, en soulignant chaque mot, que « toute la haute aristocratie, QUI NE VIENT JAMAIS AU THÉÂTRE » était là pour elle ${ }^{30}$.

La comparaison entre la province et Paris est donc permanente, la capitale demeurant cet étalon artistique à l'aune duquel tout théâtre est mesuré. Certaines villes, comme Rouen, parviennent à faire mieux encore que Paris, et Dorval s'y console de son exil forcé : «Les pièces sont parfaitement mises en scène. Antony est beaucoup mieux qu'à Paris. Les figurantes sont mises comme les rôles ${ }^{31} »$. Ce dernier détail éclaire une tare essentielle des représentations provinciales : le manque d'ensemble causé par la disparate des costumes, des décorations et du jeu d'acteurs. À Toulouse, Dorval se félicite d'avoir eu une « soirée de Paris tout à fait ${ }^{32}$ ». Là, comme à Montpellier, le public est enfin jugé digne d'intérêt par l'actrice. La scène provinciale devient alors un lieu d'exercice pour essayer de nouveaux rôles. L'actrice formée à l'école du vaudeville et du mélodrame s'entraîne ainsi, sans trop de risques, loin de la critique parisienne, à la déclamation des vers : dans Charles VII chez ses grands vassaux de Dumas, Dorval souhaite « essayer» sa voix, tester la résistance de ses poumons et de son larynx pendant la durée de cinq actes en vers. Le retour à Paris sera fondé sur les expériences du laboratoire théâtral de la province, même si le doute et la peur persistent: «Dans les mouvements de passion du $4^{\mathrm{e}}$ et $5^{\mathrm{e}}$ acte, $\mathrm{j}$ ' ai fait grande sensation et puis voilà tout. C'est peut-être bien pour la province, je ne sais pas pour Paris ${ }^{33}$ ».

Ce public provincial permet enfin à Dorval de mesurer la modernité des drames qu'elle interprète, tant le décalage apparaît immense entre les mœurs provinciales et la liberté morale des pièces romantiques créées à Paris. Elle éprouve ainsi l'étrange sensation d'être inconvenante au théâtre de Bourg-en-Bresse où, au-dessus d'un parterre de paysans et de domestiques, les loges sont toutes occupées par des femmes devant qui elle joue Angelo : « Et me voilà toute décontenancée de jouer mes femmes passionnées et désordonnées devant toutes ces petites filles, ces jeunes femmes et ces bonnes mamans. Il faut vraiment qu'il y ait à

\footnotetext{
${ }^{26}$ Corr., Chalon, 28 juin [1836], t. 3, p. 110.

${ }^{27}$ Corr., Toujours jeudi [23 juin 1836], Chalon 10 h. du soir, t. 3, p. 104.

${ }^{28}$ Corr., [Chalon, 24 juin 1836] Minuit, t. 3, p. 105.

${ }^{29}$ Corr., Marseille, ce 4 octobre [1836], t. 3, p. 151.

${ }^{30}$ Corr., [Toulouse] Dimanche [5 février 1837], t. 3, p. 219.

${ }^{31}$ Corr., [Rouen] Jeudi soir [29 août 1833], t.2, p. 276.

${ }^{32}$ Corr., Toulouse, lundi 23 janvier 1837, t. 3, p. 214.

${ }^{33}$ Corr., Sète, 6 mai 1837, t. 3, p. 240.
} 
dire dans ces ouvrages puisque je me sens toute honteuse en les jouant devant tant de femmes!». Mais cette remarque, éclairante pour la réception du drame romantique en province, vise surtout à célébrer les vertus de Chatterton aux yeux de son propre auteur, Vigny : avec le rôle de Kitty Bell, Dorval dit mettre « à l'aise toutes ces pauvres femmes qui n'ayant pas à rougir ne s'en laissent que mieux aller à leur émotion ${ }^{34} »$.

\section{Lettres provinciales ou adresses aux Parisiens?}

L'approche précédente tend à réduire le texte épistolaire à son unique valeur documentaire : nous avons lu dans les lettres de Dorval un témoignage sur le vif, susceptible de nous donner accès à la vie obscure des théâtres provinciaux. La valeur exceptionnelle de ce corpus tient certes à la concomitance temporelle, induite par le genre même de la lettre, entre l'écriture et l'événement décrit. Ce témoignage serait réputé d'autant plus "immédiat » que Dorval ne possèderait guère les qualités littéraires susceptibles d'élever ces textes à la littérature. La forme en serait libre et spontanée : comme le souligne Brigitte Diaz dans son étude consacrée à l'épistolaire (La Pensée nomade), la lettre «se joue des discours constitués » et devient ainsi «l'instrument idéal d'un savoir vivant ${ }^{35}$ ». Toutefois, pareille lecture est naïve et réductrice : d'une part, si Dorval n'est pas écrivain, force est de lui reconnaître une voix, elle-même médiatisée par l'écriture dramatique des rôles qu'elle endosse chaque soir. On reconnaîtra dans les lettres les inflexions sentimentales, les antithèses brutales et les périodes passionnées héritées de la langue de ses héroïnes de théâtre. Ainsi de ces deux phrases, presque la péroraison d'une tirade, adressées depuis Rouen à Vigny : «Toi c'est l'air et la lumière, toute le reste c'est la mort et la nuit. Oh crois-moi bien mon ange, si tu devais me retirer ton amour il vaudrait mieux me tuer tout de suite ${ }^{36} \gg$. On pourrait aussi s'interroger sur le degré d'originalité de ces lettres par rapport aux usages de l'époque, commandés par des manuels épistolaires très répandus ${ }^{37}$ tel le Nouveau Secrétaire Français : Dorval y avait-elle recours ? D'autre part, ces lettres n'ont guère de sens en dehors de la relation amoureuse qu'elles alimentent ; l'expérience théâtrale en province est à la fois cause de la séparation des amants et moyen de réduire la distance lorsque la muse Dorval joue, loin de Paris, le théâtre du poète Vigny.

Force est alors d'aborder ces documents en tant que textes dotés d'un certain degré de poéticité, mais aussi en tant que discours, conversation entre absents, et en tant qu'actes, mus par une volonté "performative » d'agir sur le destinataire. La lettre cherche à la fois à combler l'absence, à prouver une fidélité, à affirmer un dévouement propre à motiver le soutien et la compassion. Comme l'écrit Barthes dans Fragments d'un discours amoureux, la correspondance est souvent «une entreprise tactique destinée à défendre des positions, à assurer des conquêtes ${ }^{38} »$.

Ainsi, la précision des descriptions et l'inflation des détails matériels, lesquels fondent pour nous la valeur du témoignage, relève d'abord d'un désir de donner à voir toute sa vie à l'amant absent. Le régime rhétorique de l'hypotypose semble gouverner ces textes. « Te voilà bien curieux de détails. Voyons, qu'est ce que tu me demandes ${ }^{39}$ " déclare régulièrement l'épistolière avant d'entamer une description minutieuse des lieux, des faits, des gestes. À Bordeaux, une lettre obéit clairement à la commande du correspondant jaloux, dont le désir

\footnotetext{
${ }^{34}$ Corr., Vendredi 8 juillet [1836] (Bourg[en-Bresse]) 7 h. du matin, dans mon lit, t. 3, p. 125.

${ }^{35}$ Brigitte Diaz, L'Epistolaire ou la pensée nomade, PUF, 2002, p. 41.

${ }^{36}$ Corr., [Rouen] Jeudi soir [29 août 1833], t. 2, p. 277.

${ }^{37}$ Voir sur ce point La Correspondance. Les usages de la lettre au $19^{e}$ siècle, sous la direction de Roger Chartier, Paris, Fayard, 1999.

${ }^{38}$ Roland Barthes, Fragments d'un discours amoureux [1977], dans Euvres complètes, Paris, éditions du Seuil, 2002, t. V, p. 198

${ }^{39}$ Corr., ibid., t. 2, p. 275.
} 
possessif est avide de s'emparer du moindre détail : «Tu voudrais connaître ma loge dis-tu. Elle est grande et délabrée, froide. Ici tout est grand et froid ${ }^{40} »$. La lettre ouverte, écrite en plusieurs heures du jour ou durant plusieurs journées, se fait parfois dialogue continué : un moyen de «te faire vivre un peu ma vie ${ }^{41}$ ». Les missives peuvent même contenir les petits billets et autres poèmes d'admirateurs éperdus, remis pieusement au gardien de la fidélité de l'aimée : «[...] si on envoyait à Mme d'Hervey ou à Clotilde ou à Tisbé des déclarations d'amour Mme Dorval te les enverrait toutes en sortant de chaque ville, le veux-tu ${ }^{42}$ ? ». Pour ne pas alimenter la jalousie féroce de Vigny, Dorval prend bien soin de distinguer ici la comédienne des personnages joués.

Ce discours épistolaire a en effet valeur de preuve pour l'amant absent : la lettre est gage de fidélité, témoignage des bonnes mœurs, manifestation d'une capacité à résister au mille et une tentations de la vie de coulisses et d'auberges. La posture énonciative, mouvante comme dans toute correspondance, est souvent chez Dorval celle de l'avocat de soi-même : décrire des journées accaparées par les répétitions, ce n'est pas faire œuvre pour l'histoire, mais rassurer Vigny sur la vie sentimentale et sexuelle de l'actrice ambulante. Aussi Dorval trace-t-elle lettre après lettre un autoportrait bien subjectif de comédienne exemplaire, chaste et travailleuse, loin de toutes ces actrices dissolues qui ont fait, par exemple, grand tapage à Bordeaux : " Il paraît que Déjazet et Jenny Colon et Mlle George (dans le temps) en ont fait de toutes les couleurs dans ce même hôtel ». Il s'agit encore de corriger des comptes rendus de la presse provinciale, tel cet article compromettant de Méry paru dans Le Sémaphore de Marseille, qui suscite aussitôt de la part de Dorval un démenti : "Mon existence en province est telle qu'elle inspire de l'effroi aux plus indifférents. Je n'ai pas le temps de m'amuser rien n'est plaisir pour moi tout est nécessité et convenance ${ }^{43} »$. L'empressement du public masculin serait du reste causé par les rôles de femmes composés pour elle par Hugo, Dumas ou par Vigny lui-même : "Tu ne peux pas empêcher ni moi non plus cher Alfred que tous ces drames ne remuent toutes ces pauvres imaginations de la province ${ }^{44} »$, se défend-elle. Et d'affirmer qu'elle vient de faire sortir de sa loge le fils de la portière, lequel se prenait pour un Antony ou un Chatterton.

Cette représentation de la vie théâtrale élabore peu à peu un portrait de l'artiste en sainte et martyr. Dorval obéit à un modèle culturel et littéraire qui doit beaucoup au romantisme en général et à l'œuvre de Vigny en particulier. La vie d'actrice devient ainsi à Rouen une véritable oblation : "Le théâtre m'est insupportable. J'en veux à ce public des efforts que je fais pour lui. Et puis il est odieux pour moi de penser que je me tue pour les autres, sans profit pour moi, et que c'est là ma destinée jusqu'à ce que je meure ${ }^{45} »$. À Avignon, torturée par les crampes d'estomac, prise par des crises de vomissement en coulisse, Dorval évoque les «couronnes de martyre » et se dit être « vraiment sur la $\operatorname{croix}^{46} »$. Cette image christique est à lier à l'insistance avec laquelle Dorval se confère une responsabilité dans la recréation de Chatterton en province : elle souffre pour faire rayonner partout le chefd'œuvre. Elle devient en province, en l'absence de Vigny, ombre de l'auteur, co-auteur de l'ouvrage dont elle souligne à dessein le succès. Cette pièce de théâtre, substitut de son amant, Dorval s'y dévoue corps et âme. Ainsi, à Marseille : «[...] mais quelle peine je me suis donnée avec ces acteurs! et que j'en ai été malade tout le jour d'inquiétude ! Enfin je t'ai sauvé. J'avais des enfants charmants et un escalier fait pour moi $^{47} »$. Dans une autre lettre,

\footnotetext{
${ }^{40}$ Corr., [Bordeaux, mardi] 17 [février 1834]. Minuit, t. 2, p. 314.

${ }^{41}$ Corr., Vendredi 8 juillet [1836] (Bourg[en-Bresse]) 7 h. du matin, dans mon lit, t. 3, p. 125.

${ }^{42}$ Corr., [Dijon] mardi 21 juin [1836], t. 3, p. 100.

${ }^{43}$ Corr., Lyon, le $69^{\mathrm{b}}$ [novembre] 1836, t. 3, p. 176.

${ }^{44}$ Corr., Avignon, 28 déc[embre] 1836, t. 3, p. 202.

${ }^{45}$ Corr., [Rouen] Jeudi soir [29 août 1833], t. 2, p. 275.

${ }^{46}$ Corr., Avignon, samedi 24 déc[embre 1836], t. 3, p. 200.

${ }^{47}$ Corr., Marseille, ce 4 octobre [1836], t. 3, p. 151.
} 
elle ajoute : « J'ai fait adorer Chatterton à Marseille qu'on adorait déjà par la lecture ${ }^{48}$ », manière de se placer à égalité de mérite avec le dramaturge. L'œuvre devient ainsi sous la plume de Dorval «notre Chatterton ${ }^{49}$ ", enfant du poète et de sa muse dont l'épanouissement sur les scènes garantit la survie du couple. La plus grande fierté de Dorval, à Toulouse comme à Marseille, est ainsi d'obtenir un bel escalier, "comme celui de Paris ${ }^{50}$ ", et des enfants naturels en scène. Ou encore, à Montpellier, l'orgueil de la comédienne est satisfait de voir le public demander au directeur, avec un grand tapage, une représentation de Chatterton ${ }^{51}$. La comédienne finit par adhérer à l'image de tragédienne triomphante, martyr de son art, élaborée par son amant dans le cycle des poèmes dédiés à Dorval. Être à la hauteur du mythe forgé par le poète, telle est l'ambition de l'actrice. Elle semble jouir à distance, dans chacune de ses lettres, du regard de reconnaissance suscité chez Vigny par le récit complaisant de ses succès en scène : «[...] redemandée par toute la salle il m'a fallu subir quatre reprises d'applaudissements, plus de soixante jeunes gens sont montés sur le théâtre pour me complimenter. Cela te fait-il plaisir ? Oh mon Alfred, tout cela aurait été à ton cœur n'est-ce pas, j'en ai joui pour toi ${ }^{52} \gg$.

Cette insistance entre parfois en contradiction avec d'autres témoignages comme ceux de la presse provinciale, souvent réticente face au drame romantique. Ce décalage éclaire la part de mystification des lettres de Dorval et révèle finalement leur visée essentielle. Au-delà de l'entretien d'une relation amoureuse de plus en plus chaotique, il s'agit d'encourager Vigny à soutenir les intérêts parisiens de la comédienne. Les lettres réclament indirectement toujours plus de gestes publics de la part du poète ; elles commandent implicitement des articles de presse à la gloire de Dorval. Tel est le sens de cette missive envoyée de Rouen et datée du 29 août 1833 : «J'aurai fait une grande révolution ici, ils deviennent romantiques par moi. Ingrats qui m'abandonnez à Paris ${ }^{53}$ ! ». Comme l'a montré Simon Jeune dans plusieurs articles ${ }^{54}$, ces auto-célébrations et ces récriminations constantes de Dorval sont suivies d'effets. Le $1^{\text {er }}$ octobre 1833, quelques semaines après cette dernière lettre, la Revue des Deux Mondes publie un article consacré - chose rare dans cette revue littéraire - aux « théâtres des départements » : l'article, attribué par Simon Jeune à Vigny, fait l'éloge du goût de la province, de la compétence de la presse locale et dénonce les actrices rivales qui empêchent Dorval de revenir à Paris. Ce soutien indirect mais efficace de Vigny, commandé par les lettres de Dorval, a surtout été mené dans le journal le Vert-Vert, fondé en 1832 par Antenor Joly. En écho direct aux propos de la comédienne-épistolière, le Vert-Vert écrit ainsi le $1^{\text {er }}$ octobre 1833 : «A Arras elle fait, comme partout, une révolution littéraire ${ }^{55}$ ». D'autres correspondants que Vigny prennent du reste le relais, telle George Sand : celle-ci fait paraitre dans L'Artiste, le 17 février 1833, son article « Mlle Mars et Mme Dorval », tout à la gloire de la seconde. Ces interventions, pilotées à distance par les lettres de la comédienne, sont décisives pour la survie médiatique de Dorval, absente de la capitale.

Autrement dit, l'organisation de son retour à Paris oriente tout le discours épistolaire de Dorval sur le théâtre en province : le destinataire premier, Vigny, est parfois un écran, masquant ce tiers auquel Dorval souhaite continuer à s'adresser - le public parisien. Cela devient clair lorsque Dorval dénonce violemment depuis Montpellier « Paris-Théâtre, Paris-

\footnotetext{
${ }^{48}$ Corr., Avignon, 27 [octobre 1836] 2 h. après-midi, t. 3, p. 167.

${ }^{49}$ Corr., Toulouse, 30 janvier [1837]. Lundi, t. 3, p. 216.

${ }^{50}$ Corr., [Toulouse] Dimanche [5 février 1837], t. 3, p. 219.

${ }^{51}$ Corr., Montpellier, 11 janvier [1837], t. 3, p. 212.

${ }^{52}$ Corr., [Rouen] Mercredi 21 août [1833] 11H. 1/2 du soir, t. 2, p. 265.

${ }^{53}$ Corr., [Rouen], Jeudi soir. [29 août 1833], t. 2, p. 276.

${ }^{54}$ Simon Jeune, "Un Poète et sa Reine de Théâtre : campagnes de presse et vers de circonstance », Bulletin de l'Association des Amis d'Alfred de Vigny, n 8, année 1978-1979, p. 23-32 ; «Vigny, Dorval, Vert-Vert et La Gironde », Revue d'Histoire Littéraire de la France, janvier-février 1980, p. 27-59.

${ }^{55}$ S. Jeune, « Vigny, Dorval, Vert-Vert et La Gironde », article cité, p. 29.
} 
Hugo, Paris-auteurs et journalistes ${ }^{56} \gg$. La déclaration, pathétique dans sa violence, est adressée autant à Vigny qu'à cette capitale qui l'a contrainte à l'exil. Le discours sur la province ne trouve finalement son sens que dans cette « adresse » parisienne qui décide de sa forme et de son contenu. Le tableau de la vie provinciale peint par Dorval est gouverné par une seule « ligne de fuite »: Paris, l'Odéon et la Comédie-Française.

Olivier Bara, université Lyon 2, UMR LIRE, CNRS-Lyon 2

\footnotetext{
${ }^{56}$ Corr., [Montpellier] Dimanche 23 avril [1837], t. 3, p. 238.
} 